\title{
Fibroblast growth factor receptor 1 (FGFR1) amplification is a potential therapeutic target in small-cell lung cancer
}

\author{
Anne M Schultheis ${ }^{1,2}$, Marc Bos ${ }^{2,3}$, Katja Schmitz ${ }^{1,4}$, Lea Wilsberg ${ }^{1}$, Elke Binot ${ }^{1}$, \\ Jürgen Wolf ${ }^{2,3}$, Reinhard Büttner ${ }^{1,2}$ and Hans-Ulrich Schildhaus ${ }^{1,2,4}$ \\ ${ }^{1}$ Institute of Pathology, University Hospital Cologne, Cologne, Germany; ${ }^{2}$ Center for Integrated Oncology \\ Cologne/Bonn, Cologne, Germany; ${ }^{3}$ Department I of Internal Medicine, University Hospital Cologne, \\ Cologne, Germany and ${ }^{4}$ Institute of Pathology, University Hospital Göttingen, Göttingen, Germany
}

\begin{abstract}
Small-cell lung cancer (SCLC) comprises about 13-15\% of all lung cancers, and more than 29400 new cases have been diagnosed in the United States in the year 2012. SCLC is a biologically complex tumor typically occurring in heavy smokers. Its medical treatment has almost remained unchanged over the last decades and selected treatment options have not been established so far, mainly due to the lack of targetable genetic alterations. In this study we analyzed a cohort of 307 SCLC samples for fibroblast growth factor receptor 1 (FGFR1) amplification using a dual color FISH probe. FGFR1 status was correlated with clinical data. FGFR1 amplifications were observed in $5.6 \%$ of evaluable pulmonary SCLCs. Most of them $(93 \%)$ fulfilled the criteria for high-level amplification and only one case showed low-level amplification. Amplification patterns were homogenous in the entire tumor area without occurrence of any 'hot spot' areas. FGFR1 amplification status was not associated with age, sex, stage, smoking status or overall survival. FGFR1 amplification analysis by FISH analysis in SCLC is, under respect of certain technical issues, applicable in the routine clinical setting. However, the FGFR1 amplification patterns in SCLC differs strongly from the previously described FGFR1 amplification pattern in squamous cell carcinoma of the lung, as positive SCLC harbor mostly homogeneous high-level amplifications. We provide evidence that an estimated number of 1640 newly diagnosed FGFR1-positive SCLC cases in the United States annually could benefit from targeted therapy. Therefore, we recommend including SCLC in the screening for ongoing clinical trials with FGFR1 inhibitors.

Modern Pathology (2014) 27, 214-221; doi:10.1038/modpathol.2013.141; published online 26 July 2013
\end{abstract}

Keywords: FGFR1; FISH; small-cell lung cancer

Small-cell lung cancer (SCLC) remains one of the leading causes of cancer related deaths globally and represents $\sim 13-15 \%$ of all primary lung cancers. ${ }^{1}$ It typically occurs in heavy smokers, is a rapidly progressive disease and most patients present at first diagnosis with advanced stage disease. The median survival ranges from 23 months for limitedstage disease down to 8 months for extensive-stage disease. ${ }^{1}$ Over the last 30 years, medical treatment for advanced SCLC has mainly remained unchanged, consisting of a platinum-based chemotherapy regimen with optional radiation therapy depending

Correspondence: Dr H-U Schildhaus, MD, Institute of Pathology, University Hospital, Göttingen, Robert-Koch-Strasse 40, D-37075 Göttingen, Germany.

E-mail: hans-ulrich.schildhaus@med.uni-goettingen.de

Received 12 April 2013; revised 16 June 2013; accepted 17 June 2013; published online 26 July 2013 on tumor stage. ${ }^{2}$ Although targeted therapeutic options have been established for pulmonary adenocarcinomas, including EGFR (epidermal growth factor receptor) and ALK (anaplastic lymphoma kinase) inhibitors, ${ }^{1,3,4}$ and recently also for squamous cell carcinomas, ${ }^{5,6}$ small-cell carcinomas still lack therapeutically exploitable genetic alterations. So far no single molecularly targeted drug has yet shown any significant clinical activity in SCLC. However, recent advances in the molecular understanding of the oncogenic mechanisms underlying SCLC have attracted interest in the development of novel therapeutic drugs. ${ }^{2}$ In this context, our group systematically analyzed a large cohort of lung cancer specimens by conduction of 6.0 single-nucleotide polymorphism array analysis, exome sequencing, transcriptome sequencing and genome sequencing and identified pathogenetically relevant mutated genes. ${ }^{7}$ In this report, we provide evidence for 
amplification of the FGFR1 (fibroblast growth factor receptor 1) tyrosine kinase gene in SCLC.

FGFR1 is a member of the type 4 family of receptor tyrosine kinases (FGFR1-4) binding fibroblast growth factors (FGFs). Ligand-FGF-receptor interaction mediates numerous developmental programs during embryogenesis and plays a critical role in adult tissue repair and maintenance of tissue homeostasis. ${ }^{8}$ FGF binding to either of the receptors mediates signal transduction via induction of receptor dimerization and promotes a cascade of downstream signaling. FGFs and their receptors were found to have important roles in the biology of multiple cancer types. For example, FGFRs 1, 2 and 4 were found to be frequently overexpressed in breast cancer. FGFRs were also shown to mediate cell survival and motility in numerous cancer types. ${ }^{9-12}$ Promotion of oncogenesis by the FGF-FGFR signaling pathway is known to be mediated through mechanisms such as gene amplification, somatic mutations, translocations and increased expression of FGFs and/or FGFRs. ${ }^{8,9,12}$ Amplification of the FGFR1 locus at chromosome $8 \mathrm{p}$ in particular was described in several cancer types, ${ }^{12-14}$ such as breast, esophageal and head and neck carcinomas. Our group could show an oncogene dependency for a focal FGFR1 amplification in a large subset of pulmonary carcinomas, a finding that was reproduced by subsequent studies. ${ }^{5,6,15}$

Furthermore, FGFR1 amplification represents a therapeutically tractable molecular event, as a selective inhibitor of the FGFR activity caused G1 growth arrest in breast cancer cell lines. ${ }^{10}$ In SCLC, inhibition of the FGFR resulted in blockade of tumor growth, suggesting the important role of the FGF-FGFR signaling pathway for SCLC growth ${ }^{16}$ and a recent survey of potential oncogenic driver mutations also suggested FGFR1 as a potential therapeutic target. ${ }^{17}$ Different FGFR1 inhibitors are currently in phase I and II clinical trials such as BGJ 398 (Novartis), AZD 4547 (AstraZeneca), TKI 258 (Novartis), and BIBF 1120 (Boehringer-Ingelheim).

The aim of our study was to comprehensively investigate FGFR1 amplifications in SCLC. In particular, we aimed to evaluate FGFR1 amplification epidemiology and amplification patterns in SCLC and to compare it with previously described data on FGFR1 amplification in squamous cell carcinomas. The second aim of the study was to investigate whether FGFR1 fluorescence in situ hybridization on SCLC specimens is feasible in a routine clinical setting and whether this method allows to quickly and reliably identify patients for potential inclusion into ongoing clinical trials with available FGFR1 inhibitors. Furthermore we aimed to evaluate a potential correlation between clinical data and FGFR1 amplification status.

To our knowledge this is the first report of a systematic analysis of FGFR1 amplification in a large cohort of SCLCs.

\section{Materials and methods}

\section{Patients}

This study was performed with a total of 307 SCLC tumor samples with sufficient material for molecular diagnostics. The patients were screened for FGFR1 amplification in part of the routine molecular diagnostics program of the Network Genomic Medicine between January 2010 and December 2012. The Network Genomic Medicine is an academic multi center non-profit group, which provides molecular testing for personalized treatments of lung cancer patients including early clinical trials.

For analysis of patients' characteristics (age at first diagnosis, overall survival, stage and smoking status) clinical data were collected by extracting information from medical records and by questioning treating physicians as well as the patients. A complete set of clinical data was obtained for the majority of the amplified cases. However, clinical data of the non-amplified cohort were available only for a subset of patients, so that a comparable cohort was selected, resulting in an unbalanced ratio of amplified to non-amplified cases in the subgroup analysis. Baseline characteristics are displayed in Table 1. The study was conducted in concordance with the local ethical guidelines and was reviewed by the institutional ethics committee.

\section{Samples and Immunohistochemistry}

The 307 SCLC tumor samples consisted of 269 primary SCLCs, 29 lymph node metastasis and nine patients with distant metastases from SCLC (four liver metastases, one metastasis in the adrenal gland, one soft tissue, one breast, one pleura and one ovary) (Table 1).

A total of six patients with combined carcinomas consisting of small-cell carcinoma and squamous carcinoma $(n=2)$, small-cell carcinoma and adenocarcinoma $(n=3)$ or small-cell carcinoma and largecell carcinoma $(n=1)$ were included in the study, but only the small cell part was analyzed. Most samples $(n=290)$ were biopsy specimens (ie, core needle biopsies, transbronchial and lymph node biopsies) and only 17 samples were material from total tumor resection samples, including lobectomy $(n=13)$, wedge resection $(n=2)$ and lymph node extirpation $(n=2)$. No cytology specimens (ie, blocked material from fine needle aspiration) were included in the study. Tumor tissue was fixed in buffered formalin and embedded in paraffin blocks.

All primary diagnoses were reviewed by two experienced pathologists according to current recommendations. ${ }^{1}$ Thus, morphological features, eg, size of nuclei, nucleus/cytoplasm ratio, chromatin structure and nuclear molding, were evaluated on $\mathrm{H} \& \mathrm{E}$ slides. To confirm the diagnosis ancillary immunohistochemical stainings were made, eg CD56, synaptophysin, chromogranin A and cytokeratins as 
Table 1 Patients' characteristics and FISH results

\begin{tabular}{|c|c|}
\hline & $n(\%)$ \\
\hline Number of cases & 307 \\
\hline \multicolumn{2}{|l|}{ Gender } \\
\hline Male/female & $\begin{array}{c}182 / 125(59 / \\
41)\end{array}$ \\
\hline Mean age, years (range) & $65.1(33-90)$ \\
\hline \multicolumn{2}{|l|}{ Origin of specimens } \\
\hline Primary tumor & $269(88)$ \\
\hline \multicolumn{2}{|l|}{ Metastases } \\
\hline Lymph node & $29(9.4)$ \\
\hline Liver & $4(1.3)$ \\
\hline Adrenal gland & $1(0.3)$ \\
\hline Ovary & 1 \\
\hline Breast & 1 \\
\hline Pleura & 1 \\
\hline Soft tissue & 1 \\
\hline \multicolumn{2}{|l|}{ Specimens } \\
\hline Biopsy & $290(94)$ \\
\hline $\begin{array}{l}\text { Surgical tumor resection (lobectomy, wedge } \\
\text { resection, extirpation) }\end{array}$ & $17(6)$ \\
\hline \multicolumn{2}{|l|}{ FISH results } \\
\hline Non evaluable $^{\mathrm{a}}$ & $56(18)$ \\
\hline Evaluable & $251(82)$ \\
\hline Negative, $n$ (\% of all evaluable cases) & $237(94)$ \\
\hline Positive cases, $n$ (\% of all evaluable cases) & $14(5.6)$ \\
\hline High-level & $13(5.2)$ \\
\hline Low-level & $1(0.4)$ \\
\hline
\end{tabular}

Abbreviation: FISH, fluorescent in situ hybridization.

${ }^{\mathrm{a}}$ See text for explanation.

well as TTF1 (thyroid transcription factor 1). If TTF1 staining was negative, we used p63 and CK5/6 staining to exclude basaloid squamous cell carcinoma of the lung as a differential diagnosis. If appropriate or necessary, additional tests were carried out, eg, LCA (leukocyte common antigen) or CD20 staining to exclude lymphoma. Tumor diagnoses were made in accordance to the current WHO classification system. ${ }^{18}$

\section{FISH Assay}

For fluorescence in situ hybridization, three to four micron tissue sections were mounted on sialinized slides and hybridized overnight with the ZytoLight SPEC FGFR1/CEN 8 Dual Color Probe (ZytoVision, Bremerhaven, Germany). Briefly, deparaffinization, protease treatment and washes were performed on the half-automated VP2000 processor system (Abbott Molecular, Wiesbaden, Germany). After pretreatment, the slides were denatured in the presence of up to $10 \mu \mathrm{l}$ probe for $5 \mathrm{~min}$ at $75^{\circ} \mathrm{C}$ and hybridized at $37^{\circ} \mathrm{C}$ overnight. Post-hybridization SSC washes were performed at $72{ }^{\circ} \mathrm{C}$ and the slides stained with DAPI before analysis. Normal tissue including vessels, fibroblasts, lymphocytes or nontumor lung tissue served as internal positive control.
Cases were only further evaluated if control tissue nuclei displayed one or two clearly distinct signals of each color. Tumor tissue was entirely scanned for amplification hot spots by using a $\times 63$ objective and appropriate filter sets (DM5500 fluorescent microscope; Leica). If FGFR1 signals showed a homogenous distribution, random areas were used for counting the signals. Twenty contiguous tumor cell nuclei from three areas, resulting in a total of 60 nuclei, were individually evaluated with the $\times 100$ or $\times 63$ objectives by counting green FGFR1 and orange centromer 8 (CEN8) signals. As SCLC by nature often presents with overlapping cells and dense tumor areas, in some cases tumor cells from the periphery had to be chosen for reliable analysis.

FGFR1/CEN8 ratio, the percentage of cells with $\geq 5$ and $\geq 15$ FGFR1 signals and the average FGFR1 copy number per cell were calculated. Evaluation was carried out according to previously established FGFR1 amplification criteria. ${ }^{5}$ Figure 1 summarizes the evaluation algorithm.

\section{Statistics}

For statistical analysis, the SPSS software, version 20.0, (IBM Germany) was used. $\chi^{2}$-test, Fisher's exact test and $t$-test, as well as Kaplan-Meier analysis and log-rank test were used if appropriate. All tests were two-sided, with a 95\% confidence interval.

\section{Results}

\section{FGFR1 Amplification Patterns}

Having evaluated the first 100 tumors of our series, we became aware that SCLC specimens warrant a special preparation for reliable analysis of FGFR1 FISH signals. Small-cell carcinomas are morphologically characterized by a very high nucleus to cytoplasm ratio and by naturally occurring dense tumor masses with tightly localized cells and overlapping nuclei. Owing to these morphological challenges, we used at most $3-4 \mu \mathrm{m}$ thick and carefully stretched tissue slides to minimize drop out rate and optimize signal clearance.

Despite careful adaption of our slide preparation protocol, we could not gain valid data for 56 SCLC cases out of all 307 analyzed tumors (18\%; Table 1). The technical failure rate in SCLC is, therefore, higher than in pulmonary squamous cell carcinomas, which we reported recently to be $<5 \% .^{5}$ Analysis was stopped after a third ineffective attempt of evaluation. Fifty-one samples of nonevaluable cases were specimens from biopsies (ie, core needle biopsy, transbronchial and mediastinal lymph node biopsies), four samples from total lobectomy and one sample from wedge resection. All non-evaluable surgical resection samples could not be evaluated due to missing signals. Biopsy specimens, if not evaluable, lacked sufficient 


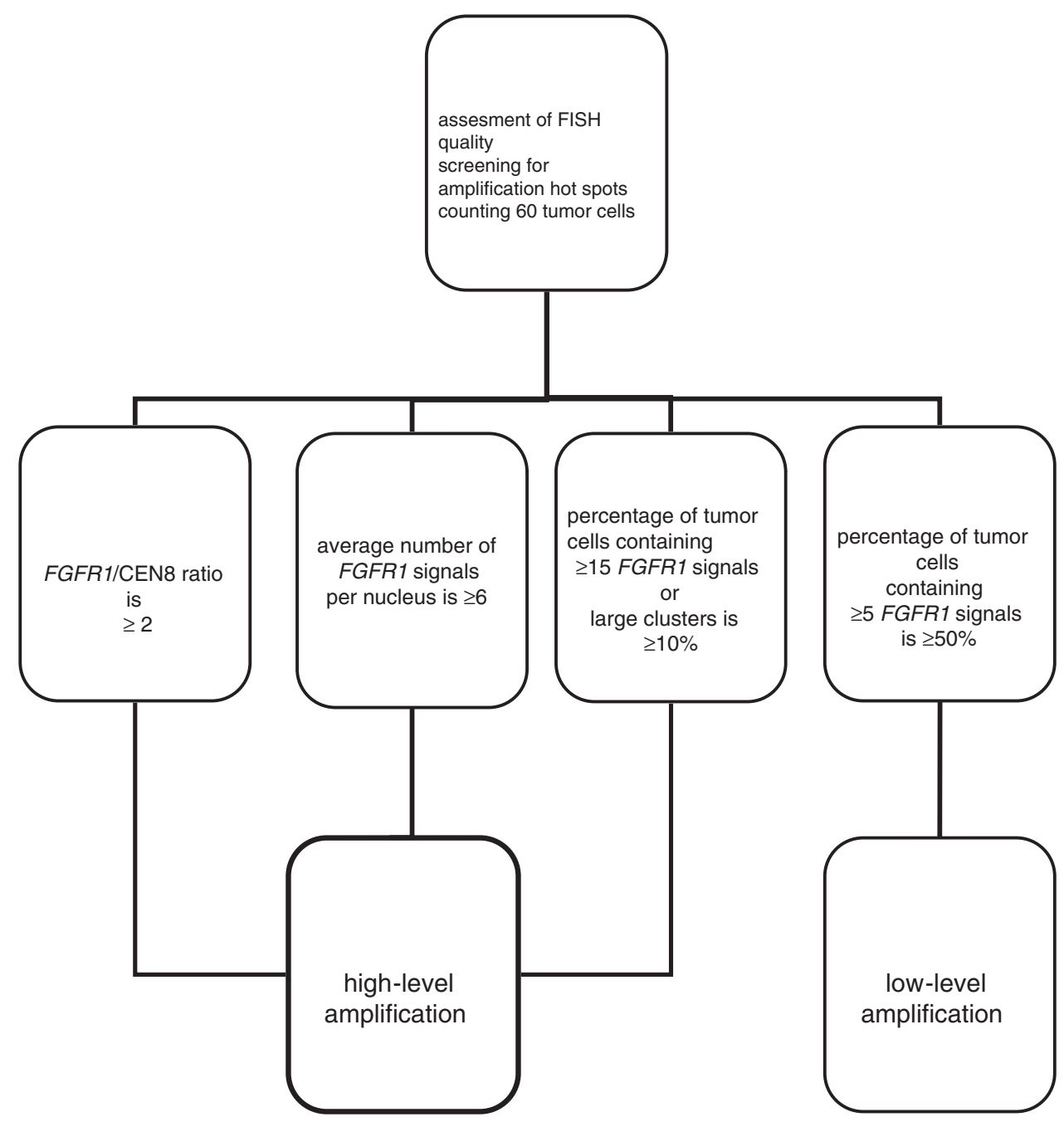

Figure 1 Algorithm for evaluation of fibroblast growth factor receptor 1 (FGFR1) status by fluorescent in situ hybridization (FISH). Highlevel amplification is defined by a FGFR1 to CEN8 ratio $\geq 2.0$ or an average FGFR1 gene count per tumor cell $\geq 6.0$ or a percentage of tumor cells containing $\geq 15$ FGFR1 gene copies of $\geq 10 \%$. Low-level amplification is defined by the absence of high-level criteria and a percentage of tumor cells containing $\geq 5$ FGFR1 gene copies of $\geq 50 \%$.

evaluable tumor cells (strong background and/or overlapping cells).

After having optimized technical issues, we found that FGFR1 signals were homogeneously distributed in all analyzed tumors. In all amplified cases, we observed an even signal distribution over the entire tumor with no 'hot spot' areas. Furthermore, all amplified tumors showed a homogenous amplification pattern with approximately the same number of FGFR1 signals in all tumor cells, with only small variations. More precisely, if for example, a tumor showed a certain cluster size, all other tumor cells would show approximately the same amplification patterns and the same cluster size with no significant variations in FGFR1 signal numbers. However, among different amplified tumors, we observed a certain variation in the signal pattern. Some amplified tumors showed mainly one or multiple smaller clusters of FGFR1 signals, whereas the amplification patterns of other tumors consisted of much higher FGFR1 signal counts per cell with larger clusters.
In one case, the tumor cell nuclei were almost completely filled with FGFR1 signals resulting in an average of 20 gene copies per cell. Table 2 shows amplification results of all FGFR1-amplified SCLCs.

Only one tumor fulfilled the criterion for lowlevel amplification, being defined by absence of criteria for high-level amplification and presence of $\geq 5$ FGFR1 signals in $\geq 50 \%$ of the tumor cells (Figure 1, Table 2).

Co-localized clusters consisting of both numerically enhanced FGFR1 and CEN8 signals did not occur. Most tumors $(n=237,94 \%$ of all evaluable tumors) were homogeneously non-amplified with 2.8 FGFR1 signals per cell on average. Polysomy, defined as average CEN8 signal count/tumor cell $\geq 3$, occurred in only $6 \%$ of all evaluable tumors. According to the reading criteria for most other FISH probes (eg, HER2 and EGFR), FGFR1 signal doublets and triplets were counted as one signal, but closely spaced groupings of signals consisting of more than three copies were considered as small clusters of 
Table 2 FGFR1 FISH-positive cases

\begin{tabular}{|c|c|c|c|c|c|c|c|}
\hline Gender & Age & \multicolumn{2}{|c|}{$\begin{array}{c}\text { FGFR1/chromosome } 8 \\
\text { signals (ratio) }\end{array}$} & \multirow{2}{*}{$\begin{array}{l}\begin{array}{l}\text { Average number of FGFR1 } \\
\text { signals per nucleus }\end{array} \\
\mathbf{7 . 6}\end{array}$} & \multirow{2}{*}{$\begin{array}{l}\text { Percentage of tumor cells containing } \\
\geq 15 \text { FGFR1 signals or clusters } \\
2\end{array}$} & \multirow{2}{*}{$\begin{array}{c}\begin{array}{c}\text { Percentage of tumor cells } \\
\text { containing } \geq 5 \text { FGFR1 signals }\end{array} \\
\mathbf{1 0 0}\end{array}$} & \multirow{2}{*}{$\begin{array}{l}\begin{array}{l}\text { Level of } \\
\text { amplification }\end{array} \\
\text { High level }\end{array}$} \\
\hline $\mathrm{F}$ & 58 & $456 / 106$ & $(4.3)$ & & & & \\
\hline M & 74 & $468 / 140$ & $(3.4)$ & 7.8 & 12 & 88 & High level \\
\hline M & 57 & $426 / 208$ & $(2.05)$ & 7.1 & 7 & 85 & High level \\
\hline M & 74 & $695 / 126$ & $(5.52)$ & 11.58 & 37 & 97 & High level \\
\hline $\mathrm{F}$ & 81 & $565 / 220$ & $(2.7)$ & 9.4 & 20 & 87 & High level \\
\hline $\mathrm{F}$ & 72 & $764 / 101$ & $(7.56)$ & 12.73 & 33 & 92 & High level \\
\hline M & 66 & $280 / 264$ & $(1.06)$ & 4.7 & 0 & 58 & Low level \\
\hline M & 57 & $427 / 168$ & $(2.6)$ & 7.12 & 5 & 75 & High level \\
\hline $\mathrm{F}$ & 73 & $383 / 87$ & $(4.4)$ & 6.38 & 8.3 & 63 & High level \\
\hline $\mathrm{F}$ & 36 & $447 / 174$ & $(2.6)$ & 7.45 & 6.7 & 90 & High level \\
\hline M & 58 & $478 / 179$ & (2.7) & 8.0 & 5 & 93 & High level \\
\hline M & 49 & $1200 / 600$ & $(2.0)$ & 20 & 100 & 100 & High level \\
\hline M & 54 & $907 / 130$ & $(7.0)$ & 15.1 & 57 & 98 & High level \\
\hline \multirow[t]{3}{*}{ M } & 73 & $503 / 104$ & (4.84) & 8.38 & 3.3 & 97 & High level \\
\hline & & Mean ratio: 3.77 & & Mean: 9.52 & Mean: 20.93 & Mean: 80.43 & \\
\hline & & Median ratio: 3.05 & & Median: 7.9 & Median: 7.65 & Median: 91 & \\
\hline
\end{tabular}

Abbreviations: F, female; FISH, fluorescent in situ hybridization; FGFR1, fibroblast growth factor receptor 1; M, male. Each line represents one FGFR1-positive tumor by displaying the individual FISH parameters. Gray highlighted numbers show the parameter fulfilling the criterion for positivity.
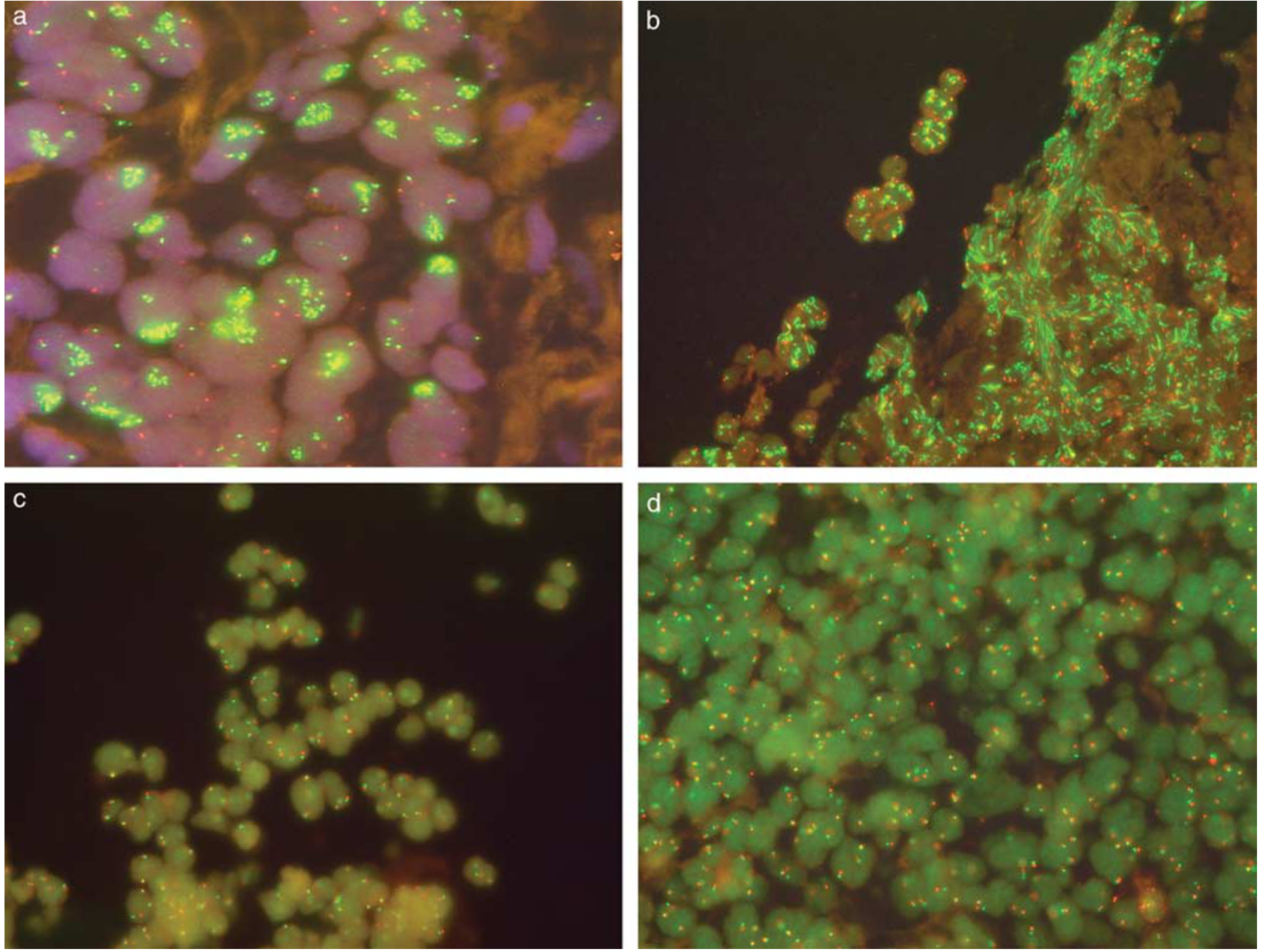

Figure 2 Fibroblast growth factor receptor 1 (FGFR1) amplification patterns. (a, b) Amplified tumors with homogenous distribution of signal patterns; note non-evaluable tumor areas due to overlapping nuclei and no clear signals (b). (c, d) Non-amplified tumors; evaluable signals adjacent to non-evaluable areas with overlapping cells.

FGFR1 signals. If clusters occurred, the minimum number of signals was in general >5 FGFR1 signals. For amplification patterns see Figure 2.
Taken together $5.6 \%(n=14)$ of all evaluable tumors $(n=251)$ showed FGFR1 amplification, of those 13 tumors reaching the criteria for high-level 
amplification $(5.2 \%$ of all evaluable tumors), and only one tumor $(0.4 \%$ of all evaluable $)$ the criterion for low-level amplification. All high-level amplified tumors showed an average number of FGFR1 signals $\geq 6$ (mean: 9.5). Seven (54\%) of these cases exhibited $\geq 10 \%$ of tumor cells containing $\geq 15$ FGFR1 signals or large clusters, and all 13 high-level amplified tumors showed a FGFR1/CEN8 ratio $\geq 2$ (Figure 1 and Table 2). One of the high-level amplified tumors showed a FGFR1 signal count of 1200. However, the FGFR1/CEN8 ratio through a quotient of $1200 / 600$ was 2.0 , being just on the border to fulfill a criterion for high-level amplification, only looking at the FGFR1/CEN8 ratio (Figure 1).

Interestingly, of all high-level amplified tumors, four tumors were negative for TTF1 immunohistochemistry. Those tumors showed significantly higher FGFR1 gene copy numbers than the other high-level amplified tumors with TTF1 positivity (mean FGFR1 gene copy number for TTF1-negative cases: 891.5, for TTF1-positive cases 461.4; $t$-test $=0.007)$.

Table 3 Median OS of FGFR1-amplified and non-amplified SCLC

\begin{tabular}{|c|c|c|c|}
\hline FGFR1 status & $\begin{array}{c}\text { Events/ } \\
\text { total }\end{array}$ & $\begin{array}{l}\text { Overall survival in } \\
\text { days }(95 \% \text { CI) }\end{array}$ & Log-rank test \\
\hline \multicolumn{4}{|l|}{ Entire cohort } \\
\hline Amplified & $3 / 13$ & Median 414 (86-738) & \\
\hline Non-amplified & $17 / 65$ & Median 387 (237-537) & $P=0.563$ \\
\hline \multicolumn{4}{|l|}{ Limited stage } \\
\hline Amplified & $1 / 3$ & Mean 142 (119-165) & \\
\hline Non-amplified & $2 / 32$ & Mean 646 (409-884) & $P=0.005$ \\
\hline \multicolumn{4}{|l|}{ Extensive stage } \\
\hline Amplified & $2 / 10$ & Median 414 (95-733) & \\
\hline Non-amplified & $15 / 33$ & Median 253 (146-360) & $P=0.109$ \\
\hline
\end{tabular}

Abbreviations: CI, confidence interval; FGFR1, fibroblast growth factor receptor 1; OS, overall survival; SCLC, small-cell lung cancer.

\section{Patient Characteristics and Correlation with FGFR1 Status}

A total of 307 patients were tested for FGFR1 amplification. Of the screened population, 182 $(59 \%)$ were male and $125(41 \%)$ were female (Table 1), which is in line with current incidence rates seen for SCLC in males and females in Europe. ${ }^{19}$ Of the amplified cases, 9 (64\%) were male and $5(36 \%)$ were female. There was no significant enrichment for any sex when comparing the amplification negative and positive patients $(P=0.75)$.

Mean age at diagnosis of the entire cohort $(n=307)$ was 65.1 years (Table 1). Age at first diagnosis could be obtained for $86 \%(n=12)$ of the amplified patients with a median of 64.5 years (range: $36-81$ ). This was not significantly different from the non-amplified subgroup $(P=0.88)$.

We acquired the smoking status of 54 patients (including 7 patients with FGFR1 amplification) of whom none were never-smokers (smoked fewer than 100 cigarettes during life time). Therefore, all patients were current smokers or had a smoking history.

We could obtain information regarding stage for a total of 78 patients including $93 \%(n=13)$ of the amplified cases. Fourty-three of these patients presented at first diagnosis with an extensive-stage and 35 with a limited-stage disease. There was no significant difference in the stage at first diagnosis between FGFR1-amplified and not amplified patients $(P=0.083)$.

Interestingly, one of the patients with a high-level amplified tumor was an only 36-year-old female, taking into account the mean age of our patient cohort of 65.1 years. At that time, she had a smoking history of 20 pack-years and died, after treatment with standard chemo-radiation therapy, 3 years after diagnosis.

\section{Survival Analysis}

We were able to obtain follow-up data for 78 patients with known FGFR1 amplification status and known
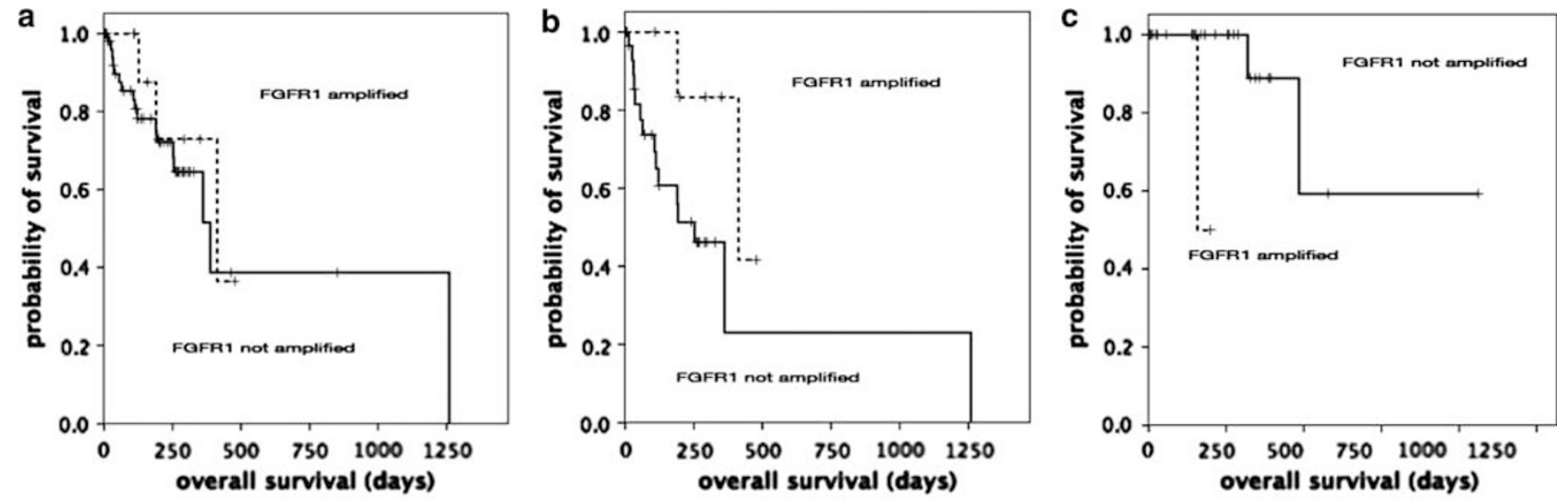

Figure 3 Kaplan-Meier Survival analysis of fibroblast growth factor receptor 1 (FGFR1) amplified vs non-amplified tumors. (a) whole cohort, (b) extensive-stage disease, (c) limited-stage disease $(P=0.005)$. 
stage at first diagnosis including 13 FGFR1-amplified cases (Table 3). The mean overall survival (OS) for patients with extensive-stage SCLC regardless of FGFR1 amplification status was 13 months (95\% CI: 4.9-21.1), and for patients with limited-stage disease the mean OS was 20.4 months (95\% CI: $12.7-$ 28.1) $(P=0.012)$. The median follow-up time of all patients was 7.3 months.

When we compared the OS of FGFR1-amplified with non-amplified patients, regardless of stage, we did not detect any significant difference between the two groups $(P=0.56)$. This held true for patients with extensive-disease at first diagnosis $(P=0.11)$ (Figure 3). Although we noticed a trend that FGFR1amplified patients with limited-stage SCLC had a worse outcome than non-amplified patients with limited-stage SCLC (log-rank test, $P=0.005$; Table 3, Figure 3). Despite this statistical significance, the clinical relevance still needs to be verified in larger studies as our cohort was small and showed a high rate of censoring.

\section{Discussion}

Therapeutically targetable FGFR1 amplification in smoking-associated human squamous cell carcinomas was recently described and occurs in about $20 \% .^{5,6,15,17}$ This is a clinically important finding since until now current therapies are very limited. So far no positive data about FGFR1 FISH analysis in SCLC have been described in the literature. In this study, we provide evidence that $5 \%$ of SCLC cases show targetable high-level FGFR1 amplification. To translate our findings into the emerging landscape of clinical trials with various FGF/FGFR inhibitors, patients eligible for treatment with currently investigated compounds must be identified reliably. In addition, molecular testing needs to be fast and reliable on generally very small and artificially altered biopsy specimens. We evaluated 307 small-cell carcinomas by a dual color FISH assay and found that $5.6 \%$ of all evaluable cases $(n=251)$ showed high- or lowlevel amplifications. The percentage is very close to data observed in a cohort analyzed by singlenucleotide polymorphism 6.0 by us $(3 / 51$, frequency $6 \%) .{ }^{7}$ We identified patterns of FGFR1 gene copy numbers that appear different from other genes. Importantly, the distribution of FGFR1 copies in small-cell carcinoma is also different from the FGFR1 pattern in other tumor entities and turned out to be homogeneous in all analyzed SCLCs. Small clusters ('microclusters'), as previously described in squamous cell carcinoma of the lung, did not appear in SCLC. Furthermore, high-level amplification is evenly distributed over tumor cells, lacking socalled 'hot-spot' areas, as seen in squamous cell carcinoma. Co-amplifications of FGFR1 and CEN8 signals do not appear. Our previously described scoring system in squamous cell carcinoma ${ }^{5}$ can be transferred to SCLC with some minor modification and thus might serve as a standardized screening tool to identify SCLC patients for ongoing and upcoming clinical trials with FGFR1 inhibitors.

Several compounds have already entered clinical trials giving precise FGFR1 diagnostics high clinical impact, and first results with a selective FGFR inhibitor have shown clinical response in squamous cell lung cancer. ${ }^{20}$ In the setting of these clinical trials, we believe that our FISH score might be helpful for identifying SCLC patients with significant increase in FGFR1 gene copy numbers. However, it still needs to be clarified by future response data derived from these studies, whether our criteria are equally good or even better predictor of response than traditional amplification criteria. Thus, our work represents a proposal for standardized screening for clinical trials. However, it appears likely that FGFR1 FISH assay is going to represent a companion diagnostic and there is a need for definition of interpretation criteria that could be implemented in the clinical practice. Having carefully assessed a large cohort of small-cell pulmonary carcinoma cases by applying our reading and evaluation strategy, we were able to divide positive cases into two types of amplification patterns. Low amplification level (as defined by absence of criteria for high-level amplification and $\geq 5$ FGFR1 signals in $\geq 50 \%$ of tumor cells) occurred only once in our cohort and accounts for $0.4 \%$ of all evaluable FISH-positive cases $(n=251)$, but $5.2 \%$ of small-cell carcinomas $(92 \%$ of FISH-positive tumors) show high-level amplification (as defined by an FGFR1/CEN8 $\geq 2.0$, or average number of FGFR1 signals per tumor cell nucleus $\geq 6$, or the percentage of tumor cells containing $\geq 15$ FGFR1 signals or large clusters $\geq 10 \%$ ). Previous data suggest that cancer cell lines with high-level amplification respond better to treatment with FGFR inhibitors, ${ }^{6}$ which was also confirmed in an independent study. ${ }^{15}$ Therefore, it may be expected that tumors with high-level FGFR1 amplification will respond better to treatment with FGFR inhibitors.

In our cohort, we did not see any statistical significance between clinical data and FGFR1 amplification status. There was no correlation with age at first diagnosis, stage, sex or smoking status. Furthermore, we did not see any differences in OS between SCLC patients with FGFR1 amplification compared with SCLC patients without FGFR1 amplification in our overall population. There was also no difference between amplified and nonamplified patients with extensive-stage disease. Patients with limited-stage disease and no amplification of FGFR1 had by trend a better OS in our patient cohort. However, our cohort of amplified tumors with a total of only 14 cases, is small, so that larger numbers will be needed to give reliable information. As mentioned initially, results from ongoing early clinical trials need to be awaited. In any case, it will be important to record amplification 
levels obtained by FISH in a reproducible manner as proposed by our score to correlate these clinical response data with amplification level. In conclusion, FGFR1 amplification might present a therapeutically targetable genetic lesion for hypothesized 1640 newly diagnosed FGFR1-positive SCLC cases in the United States annually and almost reaches the same patient number as ALK-positive adenocarcinomas of the lung ( 2000 newly diagnosed cases per year). These data indicate small-cell carcinomas should be included in FGFR1 amplification screening protocols of lung cancer.

\section{Acknowledgements}

The authors wish to thank Ellen Paggen, Theresa Buhl, Anna Sotnikov, Claudia Dorloff, Susann Zupp, Katharina Weckermann, Julia Schumann, Corinna Seidel, Nadia Massoudi and Birgit Bischof for their expert technical assistance.

\section{Disclosure/conflict of interest}

HUS has received honoraria and reimbursements from Pfizer, Abbott Molecular, Zytomed Systems and Roche Pharma and grants, honoraria and reimbursements from Novartis Oncology (all outside of this work). JW has served as an advisory board member of Novartis and AstraZeneca and has received research support from Novartis and honoraria from Novartis and AstraZeneca. The remaining authors declare no conflict of interest.

\section{References}

1 Travis WD. Update on small cell carcinoma and its differentiation from squamous cell carcinoma and other non-small cell carcinomas. Mod Pathol 2012;25 (Suppl 1):S18-S30.

2 Metro G, Duranti S, Fischer MJ, et al. Emerging drugs for small cell lung cancer-an update. Expert Opin Emerg Drugs 2012;17:31-36.

3 Shaw AT, Yeap BY, Solomon BJ, et al. Effect of crizotinib on overall survival in patients with advanced non-small-cell lung cancer harbouring ALK gene rearrangement: a retrospective analysis. Lancet Oncol 2011;12:1004-1012.

4 Soda M, Choi YL, Enomoto M, et al. Identification of the transforming EML4-ALK fusion gene in non-smallcell lung cancer. Nature 2007;448:561-566.

5 Schildhaus HU, Heukamp LC, Merkelbach-Bruse S, et al. Definition of a fluorescence in-situ hybridization score identifies high- and low-level FGFR1 amplification types in squamous cell lung cancer. Mod Pathol 2012;25:1473-1480.

6 Weiss J, Sos ML, Seidel D, et al. Frequent and focal FGFR1 amplification associates with therapeutically tractable FGFR1 dependency in squamous cell lung cancer. Sci Transl Med 2010;2:ra93.

7 Peifer M, Fernandez-Cuesta L, Sos ML, et al. Integrative genome analyses identify key somatic driver mutations of small-cell lung cancer. Nat Genet 2012;44: 1104-1110.

8 Eswarakumar VP, Lax I, Schlessinger J. Cellular signaling by fibroblast growth factor receptors. Cytokine Growth Factor Rev 2005;16:139-149.

9 Grose R, Dickson C. Fibroblast growth factor signaling in tumorigenesis. Cytokine Growth Factor Rev 2005;16: 179-186.

10 Koziczak M, Holbro T, Hynes NE. Blocking of FGFR signaling inhibits breast cancer cell proliferation through downregulation of D-type cyclins. Oncogene 2004;23:3501-3508.

11 Suyama K, Shapiro I, Guttman M, et al. A signaling pathway leading to metastasis is controlled by $\mathrm{N}$-cadherin and the FGF receptor. Cancer Cell 2002;2:301-314.

12 Brooks AN, Kilgour E, Smith PD. Molecular pathways: fibroblast growth factor signaling: a new therapeutic opportunity in cancer. Clin Cancer Res 2012;18: 1855-1862.

13 Bass AJ, Watanabe $\mathrm{H}$, Mermel $\mathrm{CH}$, et al. SOX2 is an amplified lineage-survival oncogene in lung and esophageal squamous cell carcinomas. Nat Genet 2009; 41:1238-1242.

14 Zhao X, Weir BA, LaFramboise T, et al. Homozygous deletions and chromosome amplifications in human lung carcinomas revealed by single nucleotide polymorphism array analysis. Cancer Res 2005;65: 5561-5570.

15 Dutt A, Ramos AH, Hammerman PS, et al. Inhibitorsensitive FGFR1 amplification in human non-small cell lung cancer. PLoS One 2011;6:e20351.

16 Pardo OE, Latigo J, Jeffery RE, et al. The fibroblast growth factor receptor inhibitor PD173074 blocks small cell lung cancer growth in vitro and in vivo. Cancer Res 2009;69:8645-8651.

17 Sos ML, Dietlein F, Peifer M, et al. A framework for identification of actionable cancer genome dependencies in small cell lung cancer. Proc Natl Acad Sci 2012;109:17034-17039.

18 Beasley MB, Brambilla E, Travis WD. The 2004 World Health Organization classification of lung tumors. Semin Roentgenol 2005;40:90-97.

19 Riaz SP, Luchtenborg M, Coupland VH, et al. Trends in incidence of small cell lung cancer and all lung cancer. Lung Cancer 2012;75:280-284.

20 Wolf Jurgen, LoRusso Patricia M, Camidge Ross D, et al. A phase I dose escalation study of NVP-BGJ398. Cancer Res 2012;72(Suppl 1):abstract LB-122. 